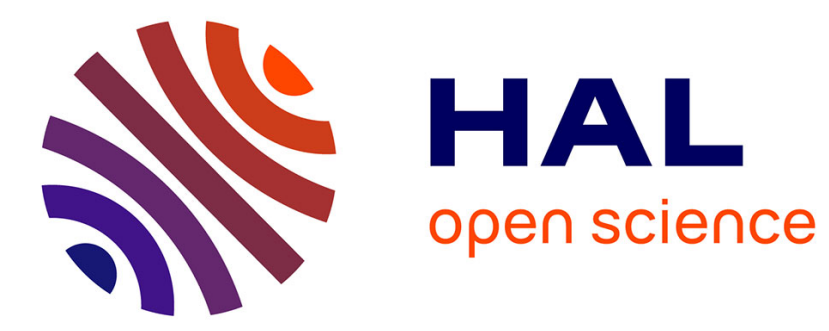

\title{
Teaching digital control of substation and IEC 61850 with a test bench validation
}

Antoine Labonne, Raphaël Caire, Thierry Braconnier, Laurent Guise, Mario Jardim, Nouredine Hadjsaid

\section{- To cite this version:}

Antoine Labonne, Raphaël Caire, Thierry Braconnier, Laurent Guise, Mario Jardim, et al.. Teaching digital control of substation and IEC 61850 with a test bench validation. IEEE Transactions on Power Systems, 2020, 36 (2), pp.1175-1182. 10.1109/TPWRS.2020.3010446 . hal-02907397

\section{HAL Id: hal-02907397 https://hal.science/hal-02907397}

Submitted on 3 Feb 2022

HAL is a multi-disciplinary open access archive for the deposit and dissemination of scientific research documents, whether they are published or not. The documents may come from teaching and research institutions in France or abroad, or from public or private research centers.
L'archive ouverte pluridisciplinaire HAL, est destinée au dépôt et à la diffusion de documents scientifiques de niveau recherche, publiés ou non, émanant des établissements d'enseignement et de recherche français ou étrangers, des laboratoires publics ou privés. 


\title{
Teaching digital control of substation and IEC 61850 with a test bench validation
}

\author{
Antoine Labonne, Raphael Caire, Senior Member IEEE, Thierry Braconnier, \\ Laurent Guise, Mario Jardim, Nouredine Hadjsaid, Senior Member IEEE
}

\begin{abstract}
This paper proposes an original Smart-Grid test bed aimed at teaching the IEC 61850 standard to students and professionals. It includes emulation of medium voltage loads and generators, with a supervisory control and data acquisition system. With this test bed, lab classes focused on the setup and configuration of protection relays using semantics of xml language are proposed. For this, GOOSE services and their functionalities are taught and tested with experiments involving real cases. The presented lab classes are part of a dedicated complete pedagogic module comprising lectures and experiments. Through the development, the test and the deployment of their own solutions in an actual distribution grid, the students learn by integrating theory and practice through the complete chain of Smart-Grid solutions: from the electrical to the communication layers.
\end{abstract}

Index Terms - Power engineering education, Distribution grid, Experimental platform, Intelligent Substation, Protection relay, Smart-Grids, Standard, IEC 61850.

\section{NOMENCLATURE}

ANR: French equivalent of US National Science Foundation ANSI: American National Standards Institute

ANSI A51: Standard dedicated to overcurrent protection BR-IEDn: Breaker of IEDn

CET850: Schneider Electric Software

CID: Configured IED Description

DNP: Distributed Network Protocol

ENSE3: Ecole Nationale Supérieure de l'Energie, l'Eau et l'Environnement

G2Elab: Grenoble Electrical Engineering Laboratory

GOOSE: Generic Oriented Object Substation Event

ICT: Information and communication technology

IEC 61850: Standard for Smart Grids

IED: Intelligent Electronic Device

IED Scout: Software of Omicron Company

LD: Logical Device

LN: Logical Node

OSI: Open System Interconnection

PTOC: Protection Time Overcurrent

PTRC: Protection Trip Conditioning

PREDIS: Production et Réseaux Energies DIStribuées

Ring LAN: Ring Local Area Network

SAS: Substation Automation System

SCADA: Supervisory Control And Data Acquisition System SCD: Substation Configuration Description
SCL: Substation Configuration Language

Sepam: Schneider Trademark (protective relays)

S84: Sepam type 84

SINARI: Sécurisation des Infrastructures et Analyse des

Risques (French research project funded by ANR)

SV: Sample Value

Wireshark: Open source Software

\section{INTRODUCTION}

CINCE the early 2000s, the world of electrical network operations has been evolving steeply. The trend is towards the decentralization of automation and to promote the interoperability of systems and electrical networks in real time. To enable this, several standards are emerging and constantly evolving [1][2]. Among them, the IEC 61850 standard [3] introduces convergence to common data models, which is the focus of this paper. In order to bring these computer models closer to real processes, a complete teaching method addressing this subject within a substation with real protection relays is proposed. Several articles address different topics of the IEC 61850 standard. Among these, K. Kaneda. et al. [4] focus on Substation Automation System and design a method around Ring LAN. Many details are found on the Logical Node family. S. Roostaee et al., [5] address peer-to-peer (horizontal) communication, GOOSE messages, and the main features of IEC 61850. Q. Song et al. [6] concentrate on two items: the analysis and comparison of pilot application and control functions. D. M. E. Ingram [7] focuses on bus protection performance with a real time digital simulator and real substation devices. They use Sample Value (SV) measurements and GOOSE at the Merging Unit to test the transformer protection relay. Finally, S. Kariyawasam et al. [8], C. Ghafari [9], A. Apostolov [10] are closer to the applications that are devoted in this article. They propose different exercises around IEC 61850 based on Substation Automation System (SAS). Their originality is in the use of real time target in the protection scheme. In the same way as the previously cited authors, practical labs based on the semantics of the IEC 61850 standard are proposed here. Compared to [7], an additional view on GOOSE messages with real components (protective relays) in the different levels (process, bay and substation) is addressed. Sample Values as [7][11] are not covered, but instead, tools such as Wireshark and IED Scout [12] are introduced in complement to courses and labs.

This work was supported in part by the Agence Nationale de la Recherche (ANR) under the grant ANR-09-SECU-11 and Schneider Electric.

A. Labonne R. Caire T. Braconnier N. Hadjsaid are with Univ. Grenoble Alpes, CNRS, Grenoble INP†, G2Elab, 38000 Grenoble, France, (e-mail: antoine.labonne@grenoble-inp.fr, raphael.caire@grenoble-inp.fr, thierre.braconnier@grenoble-inp.fr) †Institute of Engineering Univ. Grenoble Alpes

L. Guise M. Jardim are with Schneider Electric 38000 Grenoble, France (e-mail: laurent.guise@ se.com, mario.jardim@ schneider-electric.com) 
This article aims to summarize part of a set of courses presented at a Master's Degree in Grenoble INP-Ense ${ }^{3}$. All courses cover a duration of 60 hours and introduce ICT tools for Smart Grid engineers. The topics can be split into four axes:

- The Big data (10 hours of lectures and 4 hours of computer lab with Mango DB software).

- Cybersecurity (12 hours of lectures).

- The SCADA (10 hours of lectures and 4 hours of computer lab with Lynx industrial SCADA software).

- The IEC 61850 Standard (4 hours of lectures +4 hours of computer tools $+12 \mathrm{~h}$ of labs).

In this paper, only the last part of the last topic (labs on the IEC 61850 Standard) is discussed. The lab (12h) is broken down as follows: 4 hours of exchanges on IEC 61850 and 8 hours of practice using the platform presented below. The PREDIS platform [13] and technological tools are also presented to students.

\section{IEC 61850 INTRODUCTION AND SEMANTIC}

To introduce the course and labs, the Smart Grid Architecture Modeling (SGAM) is presented. Labs concern the fields indicated by the black discs in Fig. 1. The topics addressed here are Power System Equipment and Energy conversion, and feeder automation for the domain of Distribution and Transmission networks. In order to illustrate the IEC 61850 standard, Fig. 2 shows the different levels of communication from equipment to Substation. Students start to discover several protocols (oval shape in Fig. 2), generally used before IEC-61850 description, such as Modbus, DNP, Profibus, Fieldbus, and so on. In order to map the structure and connect the devices shown in Fig. 2, students use as basis the different layers of the Open System Interconnection (OSI) model proposed by the International Organization of Normalization [15]. IEC 61850 focuses on the second layer (Data Link) of the OSI description. Essentially, the labs address sections 5, 6, 7, 8 and 9 of the IEC 61850 standard (highlighted in Fig. 3).

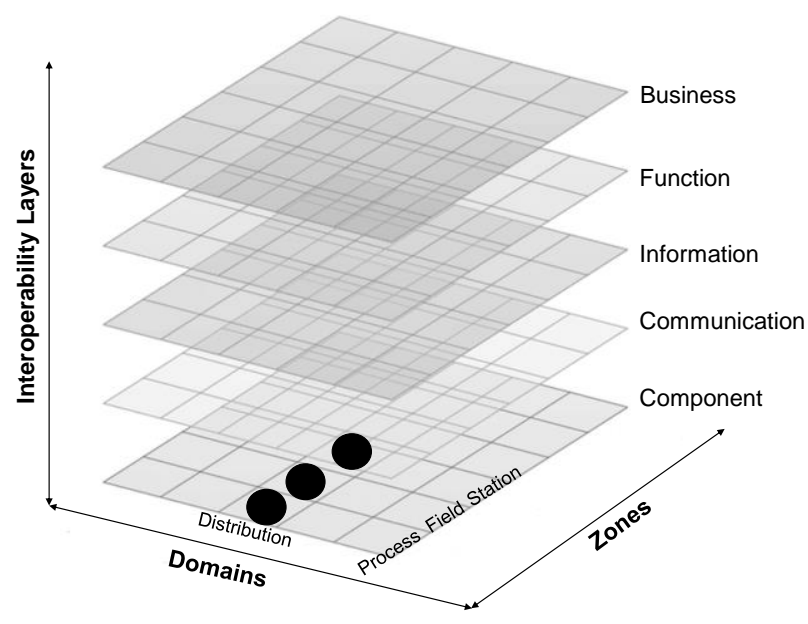

Fig. 1. Smart Grid Architecture Model. Domains and zones addressed in the course are in indicated by the black discs [14]

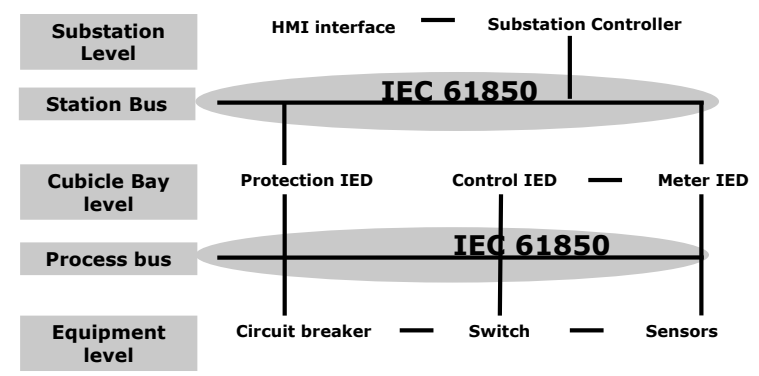

Fig. 2. Smart Substation Communication Structure using IEC-61850

61850-1: Introduction and overview

61850-2: Glossary

61850-3: General requirements

61850-4: System and project management

61850-5: Communication requirements for functions and device models

61850-6: Configuration description language

61850-7: Basic communication structure for substation and feeder equipment

61850-8, 9: Specific communication

service mapping

61850-10: Conformance testing

Fig. 3. Description of IEC 61850 chapters

\section{THE EXPERIMENTAL PLATFORM USED IN THE LAB CLASS}

All labs are tested with realistic applications on a Smart Grid Intelligent Substation (see Fig. 4). This test bed was initially developed as part of the French national funded ANR 'SINARI' Project to study the vulnerabilities that emerge between power systems and communication networks [16]. As mentioned in [16], this platform has four physical control and automation devices, a station bus and circuit breakers configured to reproduce their "in-field" performance.

TABLE I

Logical Nodes (LN) classes described in IEC 61850-7

\begin{tabular}{|c|l|}
\hline Logical Node & Group (Family) \\
\hline A & Automatic control \\
\hline C & Supervisory control \\
\hline D & Distributed energy resources \\
\hline F & Functional blocks \\
\hline G & Generic function references \\
\hline H & Hydro power \\
\hline I & Interfacing and archiving \\
\hline K & Mechanical and non-electrical primary equipment \\
\hline L & System logical Nodes \\
\hline M & Metering and measurement \\
\hline Q & Protection functions \\
\hline R & Power quality events detection related \\
\hline S & Supection related functions \\
\hline S & Instrument transformer and sensors \\
\hline W & Wind power \\
\hline X & Switchgear \\
\hline Y & Power transformer and related functions \\
\hline Z & Further (power system) equipment \\
\hline
\end{tabular}




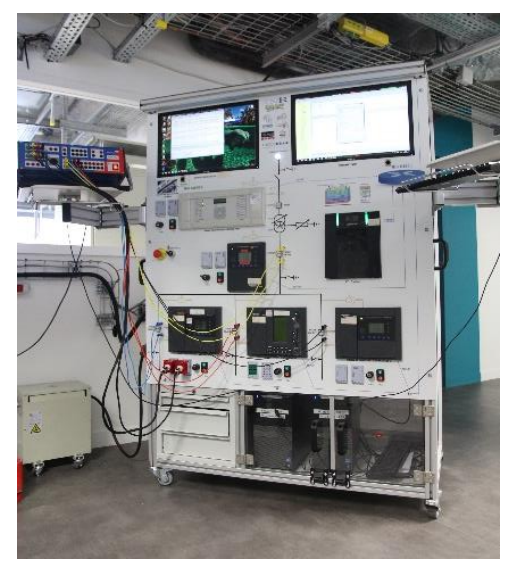

Fig. 4. Smart Grid Intelligent Substation coupled to a linear power amplifier

It could also be plugged to a real reduced scale distribution network [13]. For all the labs presented below, current generators are mainly used to test real cases. The next chapters address different experiments using advanced services, such as Generic Oriented Object Substation Event (GOOSE). The Network configuration of a Smart Grid Intelligent Substation platform is described in Fig. 5.

\section{LABS DESCRIPTION}

The course comprises five different experimental labs. The goal is to explain to the students the content of the IEC standard and the semantics of each message service for the network. It emphasizes the setting up procedures and the utilization of Generic Oriented Object Substation Event (GOOSE). Labs 0 to 4 propose several tests, including the configuration of the network and the notions addressed during the lectures. The chapter "Educational Outcomes" discusses the main results and outcomes.

\section{A. System modelling and configuration (lab 0 and lab 1)}

Many stakeholders are currently considering IEC 61850 only as a communication protocol which brings benefits for realtime operation on the field. Actually, IEC 61850 can bring much more than this and appears as a key mean along the whole life cycle of any distribution utility smart asset. It is important to let students understand that IEC 61850 can be used very efficiently with the objective to capture user requirements, thanks to a formal approach relying on IEC 61850 principles and language. This lab puts into practice the main elements that are the basis for such a top-down system approach, more specifically:

- The different namespaces (related to substation, feeder, distributed energy resources), already published or coming-up

- The three main language domains which are covered by IEC 61850: the process side (encompassing the electrical topology and associated equipment, as well as the associated functions), the functions side, and finally the communication side.

The first experimental class - lab 0 - intends to introduce a large set of examples. Different solution sections, primary and secondary devices as well as distributed functions allow the students to define the solution components, their frontiers and their functional dependencies.

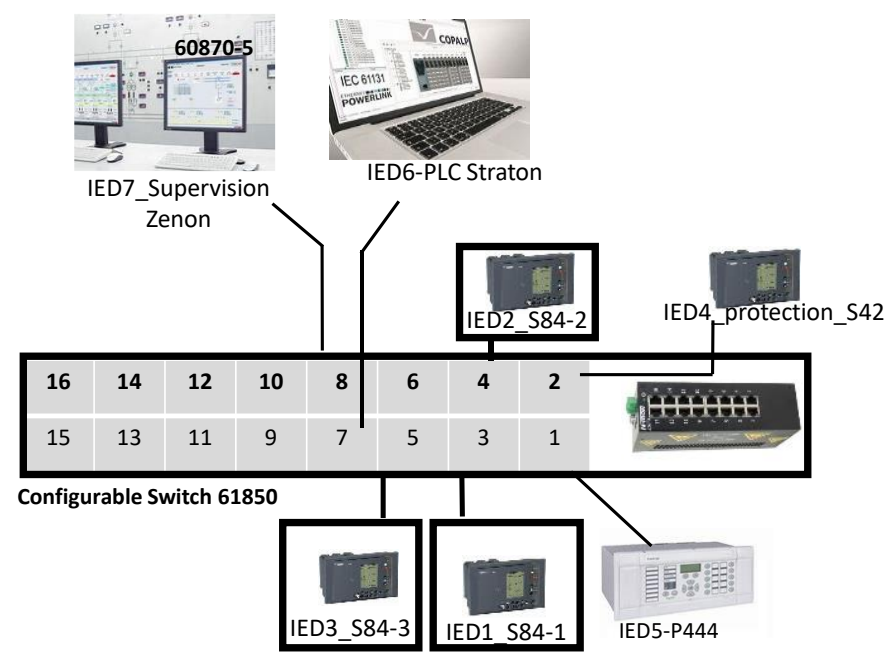

Fig. 5. Network configuration of a Smart Grid Intelligent substation

The objective of lab 1 is to configure and set up the Intelligent Electronic Devices (IED) of a Smart Grid Intelligent Substation platform. Students create CID (Configured IED Description) and SCD (Substation Configuration Description) files for the Network configuration. Fig. 6 shows a simplified configuration for all the labs corresponding to the highlighted IED. In this session, students discover the semantics of the IEC 61850 standard, re-using the knowledge acquired during the previous lab. They use Schneider Electric software (CET 850) and generate their configuration description files (CID and SCD). Then they edit these files with a text editor to understand their semantics. The SCD file shown in Fig. 7 describes the configuration of the equipment in Fig. 6.

Afterwards, students distinguish the differences between the "Substation Configuration description" and the "Configured IED description". They can then edit their own files to improve the description of the substation. In this lab, the addressing plan of devices communication is also provided. They are then able to move forward and explore all the remaining labs described in the following sections.

\section{B. GOOSE Services generation and settings (lab 2 and lab 3)}

The main objective is to use and set "GOOSE publisher/subscriber services" from IEC 61850 with Smart Grid Intelligent Substation between different Intelligent Electronic Devices (IED).

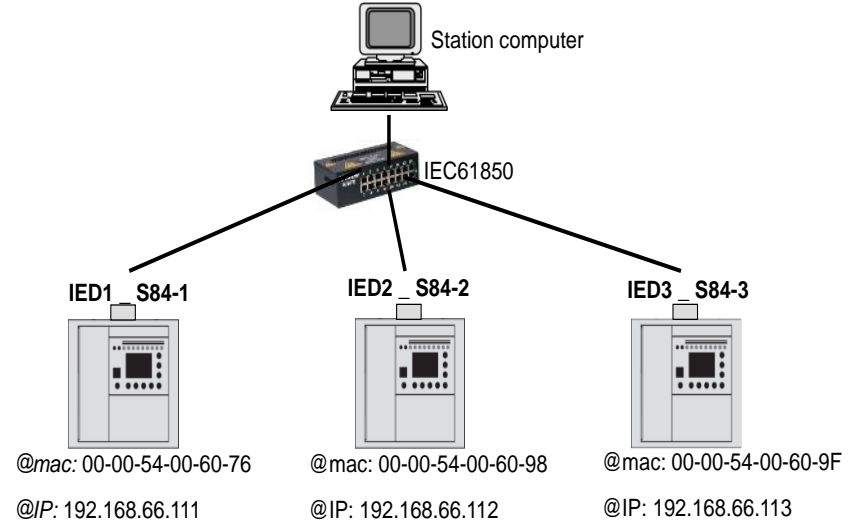

Fig. 6. Simplified IP configuration for an intelligent substation 
In this work, the focus is on IED1 (S84_1), IED2 (S84_2) and IED3 (S84_3). For all the labs on the GOOSE services generation, the organization of a GOOSE model is reminded. Students are guided through the creation of a dataset and GOOSE control block. For this, they have to closely consult the IEC 61850 Standard [3].

To illustrate the principle of the GOOSE services implementation, lab 2 (load shedding) is proposed as illustrated in Fig. 8. This is a lab designed to foster the understanding of GOOSE operation settings. It uses XCBR (switchgear and circuit breaker descriptions) as described in Table I.

The goal is to use GOOSE publisher/subscriber services to operate the devices and test this communication through the platform.

By way of example, if circuit breaker BR-IED1 must cause the opening of the other two circuit breakers (BR-IED1 and BRIED3), the procedure begins with the creation of a dataset (DS1) in an appropriate logical node of the physical device (BRIED1). The data attribute to be changed is "stVal", inside data class "Pos". Data "Pos.stVal" stands for the current state of the circuit breaker - open or closed. To be sent to other devices, a GOOSE control block (CB1) must be created, as described below. Inside the GOOSE control block, the dataset is selected and many settings may be modified (such as re-sending time for the communication). Then, students need to set up the publisher (IED 1) and the assignment of the messages for subscribers (IED2 and IED3). Students create CID and SCD files before checking the behavior on the Smart Grid Intelligent Substation platform. Thanks to this, the learners understand firstly the logic behind GOOSE services. Then, they check their functionalities for each lab. Thereafter, the use of PTRC and PTOC GOOSE services (lab 3) are presented. The aim of lab 3 (load shedding by current protection) is to compare two methods of protection (Logical Node $\mathrm{P}$ as presented in Table I).

$<$ SubNetwork name="IEC61850">

$<$ ConnectedAP apName="AP1" iedName="IED1">

$<$ Address $>$

$<$ P type $="$ IP" xsi:type="tP IP">192.68.66.111</P>

$<P$ type $="$ IP-SUBNET" xsi:type $=" t P \_I P-S U B N E T ">255.255 .255 .0</ P>$

$<P$ type $=" I P-G A T E W A Y " x$ si:type $="$ tP_IP-GATEWAY" $>0.0 .0 .0</ P>$

$<$ P type $=$ "OSI-PSEL" xsi:type $=$ "tP OSI-PSEL">00000001</P>

$<$ P type="OSI-SSEL" xsi:type="tP_OSI-SSEL">0001</P>

$<$ P type="OSI-TSEL" xsi:type="tP_OSI-TSEL">0001</P>

$<$ Address $>$

$</$ ConnectedAP $>$

$<$ ConnectedAP apName="AP1" iedName="IED2">

$<$ Address $>$

$<$ P type $=" I P "$ xsi:type="tP IP">192.68.66.112</P>

$<$ P type="IP-SUBNET" xsi:type="tP_IP-SUBNET">255.255.255.0</P>

$<$ P type="IP-GATEWAY" xsi:type="tP_IP-GATEWAY" $>0.0 .0 .0</ P>$

$<$ P type $=$ "OSI-PSEL" xsi:type="tP OSI-PSEL">00000001</P>

$<$ P type="OSI-SSEL" xsi:type="tP_OSI-SSEL">0001</P>

$<$ P type="OSI-TSEL" xsi:type $=$ "tP_OSI-TSEL">0001</P>

$<$ Address $>$

$</$ ConnectedAP $>$

$<$ ConnectedAP apName="AP1" iedName="IED3">

$<$ Address $>$

$<\mathrm{P}$ type $=" I P "$ xsi:type="tP IP">192.68.66.113</P>

$<P$ type $=$ "IP-SUBNET" xsi:type $=$ "tP_IP-SUBNET" $>255.255 .255 .0</ P>$

$<$ P type $=$ "IP-GATEWAY" xsi:type $=$ "tP_IP-GATEWAY" $>0.0 .0 .0</ P>$

$<\mathrm{P}$ type $=$ "OSI-PSEL" xsi:type $=$ "tP OSI-PSEL">00000001</P>

$<P$ type $=$ "OSI-SSEL" xsi:type $=$ "tP OSI-SSEL">0001</P $>$

$<$ P type="OSI-TSEL" xsi:type="tP_OSI-TSEL">0001</P>

$<$ Address $>$

$</$ ConnectedAP $>$

$</$ SubNetwork $>$

Fig. 7. SCD file semantics

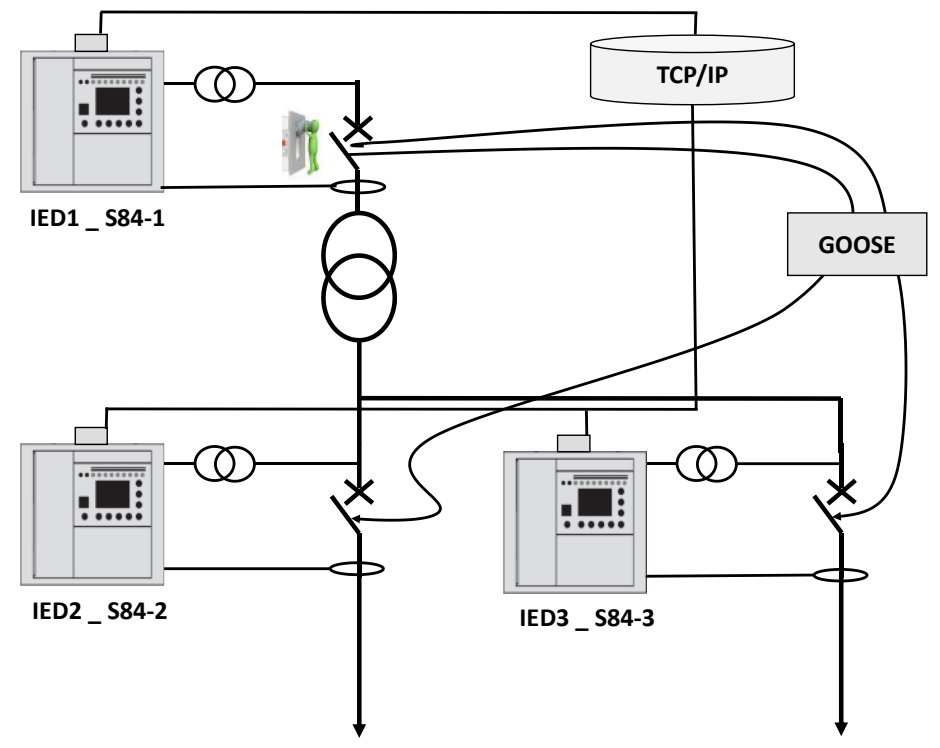

Fig. 8. Schematic description of Lab 2 TABLE II

Main settings for Smart Grid Intelligent Substation IED platform

\begin{tabular}{|l|c|c|c|}
\hline \multicolumn{1}{|c|}{ Device } & Name & IP address & MAC address \\
\hline $\begin{array}{l}\text { Protective } \\
\text { relay MV }\end{array}$ & IED1,S84_1 & 192.168 .66 .111 & 00.00 .54 .00 .60 .76 \\
\hline $\begin{array}{l}\text { Protective } \\
\text { relay MV }\end{array}$ & IED2,S84_2 & 192.168 .66 .112 & 00.00 .54 .00 .60 .98 \\
\hline $\begin{array}{l}\text { Protective } \\
\text { relay MV }\end{array}$ & IED3,S84_3 & 192.168 .66 .113 & $00.00 .54 .00 .60 .9 F$ \\
\hline
\end{tabular}

In several applications, "overload and phase-to-phase short circuit", like Protection Time over Current (PTOC, ANSI A51) and classical protections, such as Protection Trip conditioning (PTRC) are often used. Lab 2 allows us to test separately these two types of protection by using GOOSE services. Fig. 9 shows the description of Lab 3.

Following the procedures described in Lab 2, students create two others GOOSE services. The GOOSE messages allow the opening of all the breakers (BR-IED2, BR-IED3) below the secondary of the transformer when IED1 detects an overcurrent.

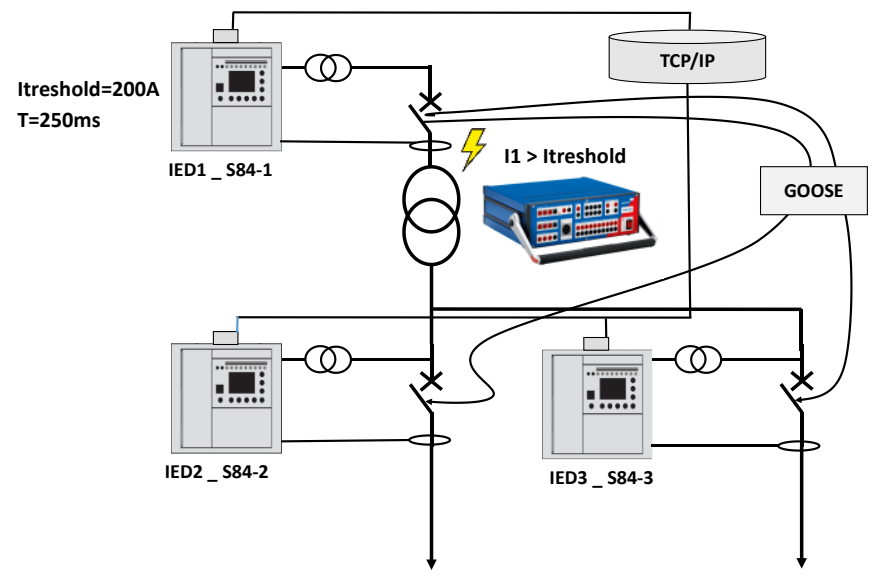

Fig. 9. Schematic description of Lab 3 
The flowchart depicted in Fig. 10 describes the steps of this lab. They set a threshold current at 200 A during a period of $250 \mathrm{~ms}$ as described in Fig. 9. Then, students set a different transforming ratio for the current transformer (250 A / $5 \mathrm{~A})$. When the breaker of IED1 (BR-IED1) is open due to a short circuit at the primary of the transformer, the GOOSE services must allow the opening of all the breakers (BR-IED2, BRIED3) at the secondary of the transformer.

TABLE III

Publisher settings and subscribers assignations

\begin{tabular}{|c|c|c|c|c|c|}
\hline \multicolumn{6}{|c|}{ IED1 (publisher) settings } \\
\hline \multicolumn{6}{|c|}{ - ${ }^{g}$ Communication } \\
\hline \multicolumn{6}{|c|}{ 一面 Substation } \\
\hline \multicolumn{6}{|c|}{$-\mathrm{C}$ s84_1 } \\
\hline \multicolumn{6}{|c|}{ - LD LDO } \\
\hline \multicolumn{6}{|c|}{$-\mathrm{GC} C \mathrm{CB} 1$} \\
\hline \multicolumn{6}{|c|}{ - DS DS1 } \\
\hline \multicolumn{6}{|c|}{ [ST] LD0.XCBR1.Pos.stVal } \\
\hline \multicolumn{6}{|c|}{ IED2 and IED3 (subscribers) assignation } \\
\hline IED Name & DA & Description & G401 & G402 & G403 \\
\hline 584 & QR & Quality & & & \\
\hline S84_1 & $\mathrm{QD}$ & Quality Dataset & & & \\
\hline S84_1 & [ST] LD0.XCBR1.Pos.stVal & & & & $\mathrm{x}$ \\
\hline
\end{tabular}

The learners use current generators for generating and analyzing voltage and current waveforms (I1 and Itreshold in Fig. 9). Before testing their configurations, they must set the transformation ratio of the current transformer to check the real current and voltage.

They compare the duration of the onset of the two GOOSE protections (PTOC and PTRC). Students must describe the periodicity of GOOSE messages and must detect the events. Then, they create CID and SCD files before comparing PTOC and PTRC.

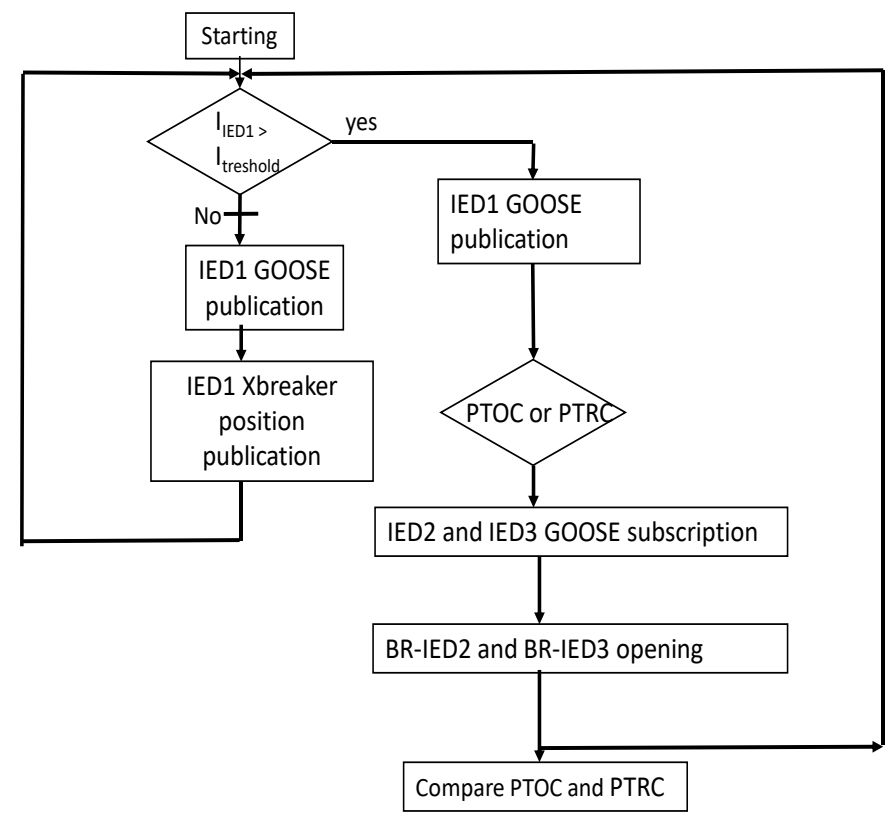

Fig. 10. Flowchart of Lab 3

\section{Representative results and analysis}

Representative results of lab 2 are presented on Fig. 11 and Table IV. Table IV aggregates the results of both strategies: PTOC and PTRC. Students use IED Scout and Wireshark in order to compare time delay and triggering analysis as shown on Fig. 11. Students summarize their results using representations such as the one shown in Fig. 12. They compare the two strategies and conclude that PTOC is faster than PTRC and that PTRC is not constrained to the delay of $250 \mathrm{~ms}$.

Current generation

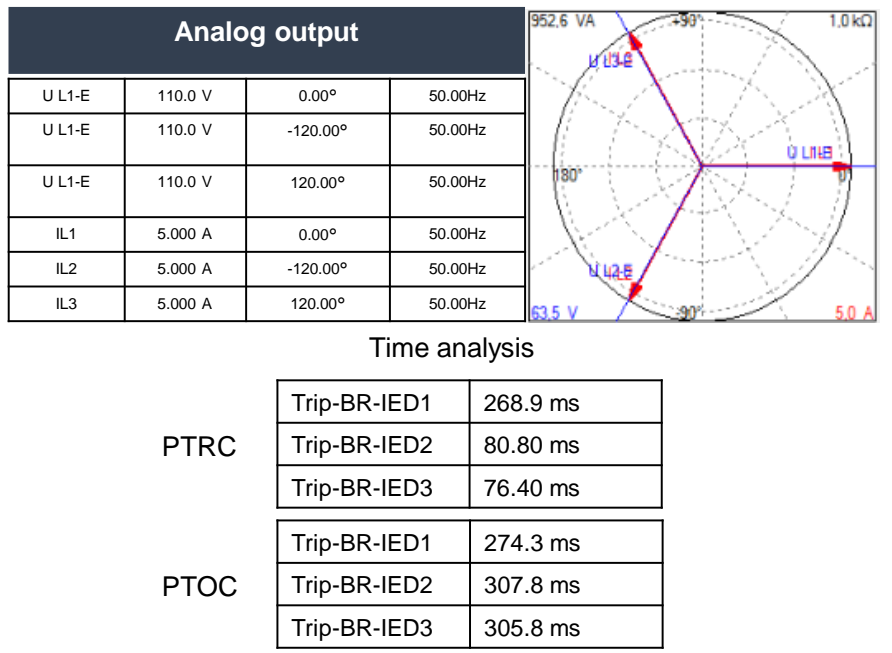

Fig. 11. IED Scout Analysis Time

TABLE IV

PTOC and PTRC description for External Trip Lab 2

\begin{tabular}{|c|c|c|c|c|}
\hline GOOSE & Publisher & Subscriber & $\begin{array}{c}\text { Overcurrent } \\
\text { triggering }\end{array}$ & Programming description \\
\hline PTOC & IED1 & IED2 IED3 & $\begin{array}{l}\text { IED1 } 24.3 \mathrm{~ms} \\
\text { IED2 } 57.5 \mathrm{~ms} \\
\text { IED3 } 55.8 \mathrm{~ms}\end{array}$ & LD0/A51.PTOC1.op.general \\
\hline TRC & IED1 & \multirow{2}{*}{$\begin{array}{l}\text { IED1 } 18.9 \mathrm{~ms} \\
\end{array}$} & LD0/PTRC1.blklnd1.stVal \\
& & IED2 IED3 & IED1.9 ms & \\
IED3 $57.5 \mathrm{~ms}$ & \\
\hline
\end{tabular}

For their assessments, students use two counter systems (Omicron CMC356) and numerical tools such as Wireshark or IEDscout (described in lab 3). At the end of lab 2, in order to emphasize the non-deterministic time for these two-overcurrent protections, Table $\mathrm{V}$ is provided to students.

TABLE V

PTOC and PTRC comparison for lab 2

\begin{tabular}{|l|c|c|c|c|c|c|}
\hline IED & $\begin{array}{c}\text { Trip } \\
\text { Time } \\
\text { PTOC } \\
(\mathrm{ms})\end{array}$ & $\begin{array}{c}\text { Standard } \\
\text { deviation } \\
(\mathrm{ms})\end{array}$ & $\begin{array}{c}\text { Vari- } \\
\text { ance }\end{array}$ & $\begin{array}{c}\text { Trip Time } \\
\text { PTRC } \\
(\mathrm{ms})\end{array}$ & $\begin{array}{c}\text { Standard } \\
\text { deviation } \\
(\mathrm{ms})\end{array}$ & $\begin{array}{c}\text { Vari- } \\
\text { ance }\end{array}$ \\
\hline 1 & 270.4 & 5.5 & 30.3 & 272.2 & 2.7 & 7.2 \\
\hline 2 & 304.5 & 6.3 & 39.2 & 77.6 & 3.2 & 10.3 \\
\hline 3 & 300.9 & 5.8 & 33.2 & 80.4 & 3.0 & 8.8 \\
\hline
\end{tabular}




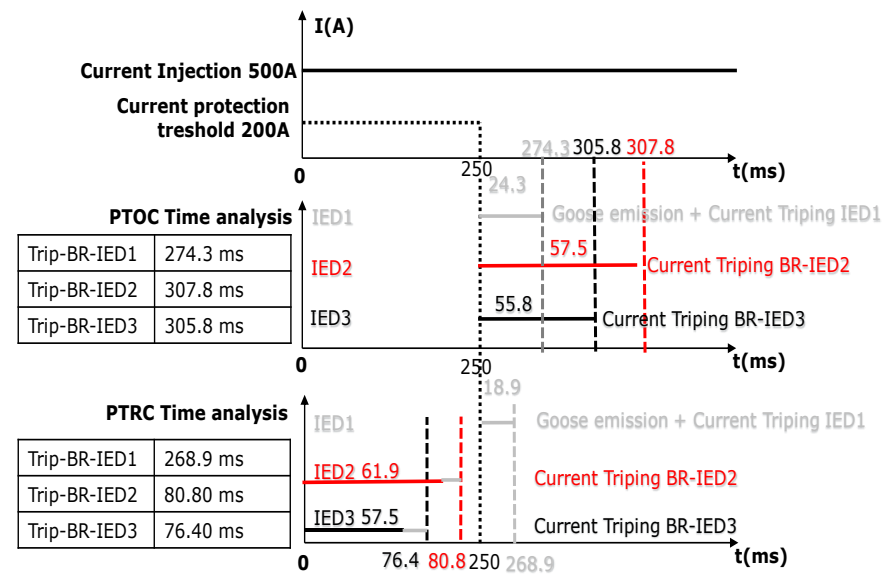

Fig. 12. Time analysis for PTOC and PTRC

\section{Sniffer and IED Scout utilities (lab 4)}

Lab 4 deals with data analysis with Wireshark and IED Scout software. In this lab, keeping the premise of lab 3, additional information on frequency, quality, power, current, among others, are added. This was done to explore the basic functionalities of two snippet software tools, IED Scout and Wireshark. IED Scout provides access to the IEDs and performs numerous useful functions when working with them. It allows the engineer to look inside the IED and at its communication. All data model and exchanged becomes visible and accessible. This is exceptionally useful for IEC 61850 devices. On the other hand, Wireshark is a network protocol analyzer that is used for advanced functions including network troubleshooting, triggers and alerts. With these sniffers, students find their GOOSE control block, their dataset and the state position of breakers. In summary, this lab gives an overview of these tools and allows students to export data for post treatment. Fig. 13 and Table VI represent two captures of these tools with IED1.

Frame 11: 104 bytes on wire, 104 bytes on interface (832 bits) Interface id : 0 ('DevicelNPF_\{5B6331E8-949D-4D7B-B78E09F55B900E8F\})

Encapsulation type : Ethernet (1)

Arrival Time : Nov 6, $201904: 49: 49.481026000$ Paris, Madrid

[Time shift for this packet : 0.000000000 seconds]

Epoch Time : 1320551389.481026000 seconds]

[Time delta from previous captured frame : 0.215342000 seconds]

[Time delta from previous displayed frame : 0.215342000 seconds] [Time since reference or first frame : 2.767552000 seconds]

Frame Number : 11

Frame Length : 104 bytes (832 bits)

Capture Length : 104 bytes ( 832 bits)

[Frame is marked: False]

[Frame is ignored: False]

[Protocols in frame : eth : ethertype :GOOSE]

[Coloring Rule Name : Broadcast]

[Coloring Rule String : eth[0] \& 1]

Ethernet II, Src : Schneide_00:60: 76 (00:00:54:00:60:76), Dst :

lec-Tc57_01:00:00

Destination : lec-Tc57 $01: 00: 00$ (01:0c:cd :01:00:00)

Source : Schneide_00:60:76 (00:00:54:00:60:76)

Type : IEC 61850/GOOSE (0x88b8)

GOOSE

APPID : 0x0000 (0)

Length : 90

Reserved 1:0x0000 (0)

Reserved $2: 0 \times 0000(0)$

goosePdu

gocbRef : IED1LD0/LLN0\$GO\$GCB1

timeAllowedtolive : 6194
datSet : IED1LD0/LLN0\$XCBR1

$\mathrm{t}$ : Jan 22, $23: 27: 35.814453125$ UTC

stNum : 16

SqNum : 23

test : False

confRev : 1

ndsCom :False

numDatSetEntries : 1

allData : 1 item

Fig. 13. Data extract from Wireshark

TABLE VI

IED Scout protocol for GOOSE services detection

\begin{tabular}{|l|l|}
\hline \multicolumn{2}{|c|}{ S84_1LD0/LLN0\$GO\$gcblab2 } \\
\hline \multicolumn{2}{|c|}{ Details } \\
\hline GOOSE control block ref. & S84_1LD0/LLN0\$GO\$gcblab2 \\
\hline Destination MAC address & $01: 0 \mathrm{C}: \mathrm{CD}: 01: 00: 00$ \\
\hline Source MAC address & $00: 00: 54: 00: 60: 76$ \\
\hline Application ID & 00 \\
\hline GOOSE ID & \\
\hline Dataset name & S84_1LD0/LLN0\$dslab2 \\
\hline VLAN ID & \\
\hline VLAN priority & false \\
\hline Needs commissioning & 2 \\
\hline Configuration revision & false \\
\hline Simulation/Test & $01: 19: 40.514$ \\
\hline Timestamp & 32 \\
\hline Status number & 0 \\
\hline Sequence number & 200 \\
\hline Time allowed to live & 2 \\
\hline Number of Dataset entries & \\
\hline
\end{tabular}

\section{E. Conclusions of experimental labs}

Firstly, students start understanding the value of a top-down system modelling and then work on the network configuration of an Intelligent Smart Grid Substation. They generate CID and SCD files and use these for all the labs by downloading their configuration data into the platform. Secondly, they address advanced messages publisher/subscriber services (GOOSE) and ensure their deployment in practical labs (lab 1).

Then labs 2 and 3 allow students to use different tools with real application cases, like overcurrent protection. For this purpose, students use hardware and software configuration tools. Lab 4 explains basic functionality of two snippet software tools. These tools analyze the communication and describe the decoding message of a GOOSE. Students change the settings of the communication and then observe the datasets and the data exchange within the messages.

\section{EDUCATIONAL OUTCOMES}

Thanks to the learning by doing strategy, these lab classes enable students to achieve four main intended learning outcomes which would not be fully possible with other classical pedagogical approaches such as lectures or programming only. At the end of the lab classes, students are capable to:

- Use and understand IEC 61850 modelling capabilities for supporting a top-down engineering process

- Use and understand the IEC-61850 semantics of for Intelligent Electronic Devices and the main communication services principles

- Program different IED and communication services

- Analyze and interpret real traffic data issue from the electrical network 
The first item is evaluated through multiple-choice questions. This evaluation gathers twenty questions and accounts $25 \%$ of the final grade. This outcome is considered as achieved when students answer correctly at least $50 \%$ of the examination questions. Then, students should be able to identify components (sensors, protective relays and communication protocols), name them and describe their functions. The second outcome is evaluated continuously throughout the experimental labs described in this paper. This evaluation accounts for $50 \%$ of the final grade. Students are evaluated on the programming and the obtained results in groups of two or three. They are oriented to check their implementations by downloading and running them on the platform. The learners must then explain the steps taken to instructors. Finally, for the last outcome (25\% of the final grade), they have to summarize and comment the results of their analyses.

\section{CONCLUSIONS AND PERSPECTIVES}

This article proposes an original Smart Grid lab class as a new way to address one key issue of the complex and large concept of Smart Grid with experimental and theoretical approaches of interoperability. Students solve real-life examples related to the operation of a distribution and power substation network by using the Standard IEC-61850. The article focuses on a small part of this standard to teach the semantics of it by programming GOOSE services. Engineers who follow this course will be aware of the association between industrial devices and sensors in a real scenario. As a result of this course, about $87 \%$ percent of the students were able to autonomously develop, implement and test their own protection strategies in real life scenarios. Protection-related aspects were prioritized since these are central for the long-term operation of the grid. Nonetheless, further use-cases could be explored in the future, such as traffic modeling, interoperability of systems as well as other services and other standards.

\section{ACKNOWLEDGMENT}

The authors thank Schneider Electric for the technical support and contribution and Dr Diego Pereira Botelho for his helpful advice and comments.

\section{REFERENCES}

[1] Y. Pradeep, P. Seshuraju, S. A. Khaparde, V. S. Warrier, S. Cherian, "CIM and IEC 61850 integration issues: Application to power systems," presented at the IEEE Power Energy Society General Meeting, Calgary, Canada, July 2009, pp. 1-6.

[2] P. Bredillet, E. Lambert, E. Schultz, "CIM, 61850, COSEM standards used in a model driven integration approach to build the smart grid service oriented architecture," presented at the First IEEE International Conference on Smart Grid Communications, Gathersburg Maryland, USA, 2010, pp. 467-471.

[3] IEC standard for Communication networks and systems for power utility automation - Part 6: Configuration description language for communication in electrical substations related to IEDs, IEC 61850-6, Dec 2009.

[4] K. Kaneda, S.Tamura, N. Fujiyama, Y. Arata, H. Ito, "IEC61850 based substation automation system," presented at the Conference POWERCON, New Delhi, India, 2008, pp. 1-8.
[5] S. Roostaee, R. Hooshmand, M. Ataei, "Substation automation system using IEC 61850," presented at the 5th International Power Engineering and Optimization Conference, Malaysia, June 2011, pp. 393-397.

[6] Q. Song, W. Sheng, L. Kou, D. Zhao, Z. Wu, H. Fang, "Smart substation integration technology and its application in distribution power grid." in CSEE Journal of Power and Energy Systems, vol 2, no 4, pp. 31-36, Dec. 2016.

[7] D. M. E. Ingram, P. Schaub, R. R. Taylor and D. A. Campbell, "SystemLevel Tests of Transformer Differential Protection Using an IEC 61850 Process Bus," in IEEE Transactions on Power Delivery, vol. 29, no. 3, pp. 1382-1389, June 2014.

[8] S. Kariyawasam, A. Wickremasuriya and A. Rajapakse, "Teaching IEC 61850 based substation automation through hands-on experiences," presented at the IEEE Electrical Power and Energy Conference (EPEC), Saskatoon, 2017, pp. 1-6.

[9] C. Ghafari. "Innovative numerical protection relay design on the basis of sampled measured values for smartgrids" $\mathrm{PhD}$ disertation Université Grenoble Alpes, France, 2016.

[10] A. Apostolov, "Multi-agent systems and IEC 61850," presented at the IEEE Power Engineering Society General Meeting, Montreal, 2006, .1-6.

[11] J. Tan, V. Green and J. Ciufo, "Testing IEC 61850 based multi-vendor substation automation systems for interoperability," presented at the IEEE PES Power Systems Conference and Exposition, Seattle, 2009, pp. 1-5.

[12] Q. Fu, J. Chen, "Design of experiment platform for digital substation based on IEC 61850," presented at the 5th International Conference on Computer Science and Network Technology, 2016, pp. 4-8.

[13] M.C. Alvarez-Hérault, A. Labonne, S. Touré, T. Braconnier, V. Debusschere, and al, "An Original Smart-Grids Test Bed to Teach Feeder Automation Functions in a Distribution Grid", in IEEE Transactions on Power System, vol 33, no 1, pp. 373-385, Jan. 2018.

[14] CEN-CENELEC-ETSI Smart Grid Coordination Group Methodologies to facilitate Smart Grid system interoperability through standardization, system and testing SG-CG/M490/I_Smart Grid Interoperability, 2014.

[15] ISO Systèmes de traitement de l'information - Interconnexion de systèmes ouverts - Modèle de référence de base,ISO 7498-2,1989.

[16] F. Planchon, E. Jehano, J. Sanchez, R. Caire "CYPRES: A Security Companion for IEC 61850 Automated Substations," presented at MATPOST, 2015, Lyon, France.

\section{BIOGRAPHIES}

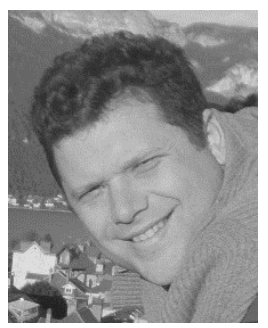

Antoine Labonne received the Master's degree in mechatronics from the University of Blaise Pascal, Aubiere, France, in 2005, and the Electrical Engineering Diploma from Grenoble Institute of Technology, Grenoble, France. Since 2005, he has been an Engineer at Grenoble INP doing his research at the G2Elab. His research interests have concerned the electrical network field, energy production systems, and renewable energy integration.

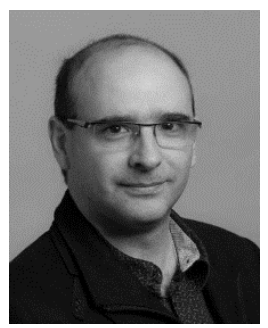

Raphael Caire (SM'00, M'04, SM'14) received the Ph.D. degree from Grenoble Institute of Technology (Grenoble INP), Grenoble, France, in 2004. He had been working in the power electronic field in the Center of Power Electronic System, USA, in 2000. In 2004 and 2005, he was a Postdoctoral Researcher in the EIfER Institute, University of Karlsruhe, Germany, on technical aspects of virtual power plant in distribution network. Between 2005 and 2006, he was with EDF R\&D as a Research Engineer. Since 2006, he has been an Associate Professor at Grenoble INP, doing his research part at the G2Elab and his teaching duty at the Ecole Nationale Superieure de l'Energie, de l'Eau et de l'Environnement. His recent works deal with new architectures of distribution networks, reconfiguration, protection, and new power electronic devices to control power flows. 


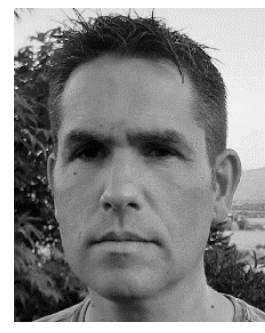

Thierry Braconnier received the Licence degree in electric distribution and automatisms from Université Grenoble Alpes, France, in 2011. Since 2011, he has been an Engineer at Grenoble INP, doing his research at the G2Elab. His research interests have concerned the electrical network field and energy production systems.

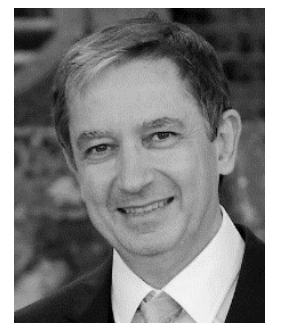

Laurent Guise, graduated from the Ecole Supérieure d'Electricité (ESE SUPELEC Engineering school) in 1981 has been working for Schneider Electric in Electrical network protection, monitoring and control system for more than 30 years. Within SchneiderElectric, Laurent Guise is awarded as Master expert in Smart Grids and IEC 61850. He is leading, at Corporate level the MV Energy influence policy (including standardisation and regulation), as well as some new innovative offering. L. Guise leads the "Roadmap" group (WG3) of the IEC System Committee on Smart Energy. He is also convening the IEC TC 57 WG17 group in charge of communication and data models for Distributed Energy Ressources integration, by extending the leading IEC 61850 standard. Within IEC TC57 he also leads the digitalisation of IEC 61850 series, which induced the setting up with the IEC Central Office of some new innovative deliverables. At European he also chairs the CENCENELEC-ETSI Co-ordination Group on Smart Energy At France level, Laurent Guise leads the AFNOR experts commission related to Smart Grids standardisation, mirroring both the IEC System committee and the CENCENELEC-ETSI Co-ordination Group on Smart Energy.

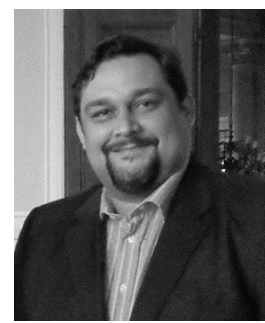

Mario Roberto Monticelli da Silva Jardim, Mechatronic engineer graduated in 2000, MBA in 2011. Has been working with systems engineering for electrical power systems since the year 2000. Is an experienced professional on the domain of supervision, protection $\&$ control of electrical grids. $\mathrm{He}$ is currently focusing on the cyber security challenges of digital systems for energy distribution. and is part of WG3 group of the IEC System Committee on Smart Energy, WG15 of IEC TC 57, IEEE PSCC working groups, and co-chair of ECSO WG1.

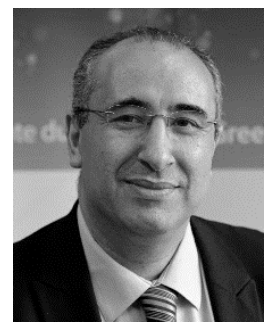

Nouredine Hadjsaid received $\mathrm{PhD}$ and the "Habilitation à Diriger des Recherches" degrees from Grenoble Institute of Technology in 1992 and 1998 respectively. $\mathrm{He}$ is presently a full professor at Grenoble INP, engineering institute of UGA, where he conducts research at G2Elab. His main expertise is in the area of "Smartgrids". He has directed the common academia-industry research center between EDF, Schneider Electric and G2Elab (IDEA: Inventer la Distribution Electric de l'Avenir) on smartgrids from 2001 to 2013. He is presently the Director of the power Engineering lab G2Elab, the Director of an ENEDIS Industrial chair of excellence on "Smartgrids" and the Chairman of Scientific Council of Think SmartGrids France. At the international level, he is presently the treasurer of IEEE Power Energy Society, and served as the vice-chair of IEEE IGETCC (Intelligent Grid and Emerging Technologies Coordination Committee) and the French representative at International Energy Agency for ISGAN-SIRFN Annex. He was the general conference chair of IEEE PowerTech'2013 held in Grenoble-France and IEEE SG4SC (SmartGrids for SmartCities) held in Paris in 2016. Dr. Hadjsaid has published more 250 scientific papers in international referred journals and conferences, has author/co-authored and directed 7 books about power and Smartgrids. 